\title{
The unquenched quark model and its last application in baryons.
}

\author{
Hugo Garcia-Tecocoatzi* \\ INFN Sezione di Genova \\ E-mail: hgarcia@ge.infn.it
}

In this contribution, we briefly analyze the formalism of the unquenched quark model (UQM) and its application to calculate the mass shifts of ground-state octet and decuplet baryons due to the coupling to the meson-baryon continuum. We describe the electro-production of Baryon-Meson states from proton in the framework of the UQM. Finally, we discuss the strangeness suppression factor within the UQM. The theoretical results are in good agreement with the values extracted from CERN and JLab experiments.

The European Physical Society Conference on High Energy Physics

5-12 July

Venice, Italy

${ }^{*}$ Speaker. 


\section{INTRODUCTION}

At the moment, the number of known light-quark mesons is much larger than the number of known baryon resonances [1]. However, it is known that the baryon spectrum is much more complex than the meson one. For instance, it is difficult to identify those high-lying baryon resonances that are only weakly coupled to the $\mathrm{N}$-channel, since they cannot be seen in elastic N-scattering experiments. In this contribution, first we present the formalism of the unquenched quark model (UQM), which is an extension of the constituent quark model, and we apply the UQM formalism to compute of the self-energies correction to the mass of ground state octet and decuplet baryons [2]. Finally, we discuss one of the latest applications of the UQM to describe the strangeness suppression in the electro-production of resonances from proton [3] within the UQM framework.

\section{UNQUENCHED QUARK MODEL}

The unquenching of the quark model for mesons $[4,5,6,7]$ and for baryons $[8,9,11]$ is a way to take into account the continuum-coupling effects. Above threshold, these couplings lead to strong decays and below threshold, they lead to virtual $(q q q-q \bar{q})$ components in the hadron wave function and shifts of the physical mass with respect to the bare mass. In the unquenched quark model for baryons $[8,9,11]$, the baryon wave function is made up of a zeroth order qqq configuration plus a sum over the possible higher Fock components, due to the creation of ${ }^{3} P_{0} q \bar{q}$ pairs, thus we have,

$$
\left|\psi_{A}\right\rangle=\mathscr{N}_{A}\left[|A\rangle+\sum_{B C l J} \int d \vec{K} k^{2} d k|B C, l, J ; \vec{K}, k\rangle \frac{\left\langle B C, \ell, J ; \vec{K}, k\left|T^{\dagger}\right| A\right\rangle}{\Delta E_{A \rightarrow B C}(k)}\right],
$$

where $T^{\dagger}$ is the ${ }^{3} P_{0}$ operator that has been recently applied to the strong decays [10].

\section{THE SELF-ENERGIES CALCULATION}

Here we present the formalism to compute the self-energy corrections within the UQM. As widely discussed in the literature, continuum effects may be extremely important, especially in the description of states close to open flavor (and, sometimes also hidden-flavor) decay thresholds. In the present work [2], the physical mass of a baryon $M_{A}$ can be written as

$$
M_{A}=E_{A}+\Sigma\left(M_{A}\right)
$$

where $E_{A}$ is the bare mass and

$$
\Sigma\left(M_{A}\right)=\sum_{B C} \int_{0}^{\infty} k^{2} d k \frac{\left|M_{A \rightarrow B C}(k)\right|^{2}}{M_{A}-E_{B C}(k)},
$$

the self-energy correction. The mass shifts are calculated by taking the bare energies as free parameters fitted to the reproduction of baryon physical masses. The values of the intermediate-baryon and -meson masses are taken from the PDG [1]. We take the contributions to the self-energies of ground state octet and decuplet baryons in our calculations. Our results are presented in the Table 1 . 
Table 1: Self-energy corrections $\Sigma\left(M_{A}\right)$ (in $\mathrm{GeV}$ ). The bare energies $E_{A}$ are obtained from Eq. (3.1).

\begin{tabular}{ccccc}
\hline Baryon & $J^{P}$ & $\Sigma\left(M_{A}\right)$ & $E_{A}$ & $M_{A}$ \\
\hline$N$ & $\frac{1}{2}^{+}$ & -0.377 & 1.316 & 0.939 \\
$\Lambda$ & $\frac{1}{2}^{+}$ & -0.359 & 1.475 & 1.116 \\
$\Sigma$ & $\frac{1}{2}^{+}$ & -0.346 & 1.541 & 1.195 \\
$\Xi$ & $\frac{1}{2}^{+}$ & -0.327 & 1.645 & 1.318 \\
\hline$\Delta$ & $\frac{3}{2}^{+}$ & -0.410 & 1.642 & 1.232 \\
$\Sigma^{*}$ & $\frac{3}{2}^{+}$ & -0.394 & 1.777 & 1.383 \\
$\Xi^{*}$ & $\frac{3}{2}^{+}$ & -0.376 & 1.908 & 1.532 \\
$\Omega$ & $\frac{3}{2}^{+}$ & -0.374 & 2.046 & 1.672 \\
\hline
\end{tabular}

\section{BARYON ELECTRO-PRODUCTION AND STRANGENESS SUPPRESSION}

The UQM wave function can be tested in the production ratios of baryon-meson states [3]. In Ref. [3] was shown that the production rates can be expressed as the product of a spin-isospin factor and a radial integral. For instance:

$$
\frac{P\left(\Lambda K^{+}\right)}{P\left(n \pi^{+}\right)}=\frac{27}{50} \frac{I_{N \rightarrow \Lambda K}}{I_{N \rightarrow N \pi}}, \quad \text { with } \quad I_{A \rightarrow B C}=\int_{0}^{\infty} d k \frac{k^{4} \mathrm{e}^{-2 F^{2} k^{2}}}{\Delta E_{A \rightarrow B C}^{2}(k)} .
$$

Here, the energy denominator represents the energy difference between initial and final hadrons calculated in the rest frame of the initial baryon A. By using the UQM, we can determine the ratios for exclusive two-body production in a straightforward way, and Table 2 shows that the experimental rates recently measured at Jlab [12] are reproduced very well by our calculation.

Table 2: Comparison between theoretical ratios of baryon-meson electroproduction from neutron and proton and experimental data. In the third column are the theoretical results of the first and second columns. Experimental data is just for proton.

\begin{tabular}{cccc}
\hline \hline $\begin{array}{c}\text { Ratio } \\
\text { from neutron }\end{array}$ & $\begin{array}{c}\text { Ratio } \\
\text { from proton }\end{array}$ & UQM & $\begin{array}{c}\text { Exp. [12] } \\
\text { from proton }\end{array}$ \\
\hline & & & \\
$\Lambda K^{0} / p \pi^{-}$ & $\Lambda K^{+} / n \pi^{+}$ & 0.227 & $0.19 \pm 0.01 \pm 0.03$ \\
$\Lambda K^{0} / n \pi^{0}$ & $\Lambda K^{+} / p \pi^{0}$ & 0.454 & $0.50 \pm 0.02 \pm 0.12$ \\
$n \pi^{0} / p \pi^{-}$ & $p \pi^{0} / n \pi^{+}$ & 0.5 & $0.43 \pm 0.01 \pm 0.09$ \\
\hline \hline
\end{tabular}

The calculation of the strangeness suppression factor, takes into account all channels involving pseudoscalar mesons in combination with octet and decuplet baryons. The results are presented in Table 3. 
Table 3: The production ratios of the $q \bar{q}$ probabilities and $\lambda_{s}$ inferred from sea quarks of the nucleon. The column $\mathrm{UQM}^{(1)}$ contains the $q \bar{q}$ production ratios with $\pi, K, \eta$ and $\eta^{\prime}$ mesons, while the column $\mathrm{UQM}^{(2)}$ only takes into account $\pi$ and $K$ mesons.

\begin{tabular}{ccccc}
\hline \hline \multicolumn{5}{c}{ Proton } \\
\hline Ratio & $\mathrm{UQM}^{(1)}$ & $\mathrm{UQM}^{(2)}$ & Exp. & Ref. \\
\hline$s \bar{s} / d \bar{d}$ & 0.265 & 0.245 & $0.22 \pm 0.07$ & {$[12]$} \\
$u \bar{u} / d \bar{d}$ & 0.568 & 0.568 & $0.74 \pm 0.18$ & {$[12]$} \\
$2 s \bar{s} /(u \bar{u}+d \bar{d})$ & 0.338 & 0.313 & $0.25 \pm 0.09$ & {$[12]$} \\
& & & $0.29 \pm 0.02$ & {$[13]$} \\
\hline \multicolumn{5}{c}{ Neutron } \\
$s \bar{s} / u \bar{u}$ & 0.265 & 0.245 & & \\
$d \bar{d} / u \bar{u}$ & 0.568 & 0.568 & & \\
$2 s \bar{s} /(u \bar{u}+d \bar{d})$ & 0.338 & 0.313 & & \\
\hline \hline
\end{tabular}

\section{CONCLUSIONS}

The observed ratios for the production of baryon-meson channels in exclusive reactions can be understood in a simple and transparent way in the framework of the UQM. Finally, the UQM value for the strangeness suppression factor in the proton is in good agreement with the value determined in exclusive reactions [5] as well as from high-energy production [13]

\section{References}

[1] C. Patrignani et al. (Particle Data Group), Chinese Physics C 40, 100001 (2016).

[2] H. García-Tecocoatzi, R. Bijker, J. Ferretti and E. Santopinto, Eur. Phys. J. A53 no.6, 115 (2017).

[3] E.Santopinto, R. Bijker, and H. García-Tecocoatzi, Phys.Lett. B759 214 (2016).

[4] J. Ferretti, G. Galatà, E. Santopinto (INFN, Genoa), Phys.Rev. D 90, 054010(2014).

[5] J. Ferretti and E. Santopinto, Phys.Rev. D 90, 094022 (2014).

[6] J. Ferretti, G. Galatà and E. Santopinto, Phys.Rev. C 88, 015207 (2013).

[7] J. Ferretti, G. Galatà, E. Santopinto and A. Vassallo, Phys.Rev. C 86015204 (2012).

[8] R. Bijker, J. Ferretti and E. Santopinto, Phys.Rev. C85, 035204 (2012).

[9] E. Santopinto and R. Bijker, Few Body Syst. 44 95-97 (2008).

[10] J. Ferretti, R. Bijker, G. Galatà, H. García-Tecocoatzi and E. Santopinto, Phys.Rev. D 94, 074040 (2016).

[11] R. Bijker and E. Santopinto, Phys. Rev. C 80, 065210 (2009)

[12] M.D. Mestayer et al. [The CLAS Collaboration], Phys.Rev.Lett. 113, 152004 (2014).

[13] G. Bocquet et al., PhysLett. B366, 447 (1996). 